\title{
Graphene sheet versus two-dimensional electron gas: A relativistic Fano spin filter via STM and AFM tips
}

\author{
A. C. Seridonio, ${ }^{1,2}$ E. C. Siqueira, ${ }^{2}$ F. M. Souza, ${ }^{3}$ R. S. Machado, ${ }^{2}$ S. S. Lyra, ${ }^{2}$ and I. A. Shelykh ${ }^{4,5}$ \\ ${ }^{1}$ Instituto de Geociências e Ciências Exatas-IGCE, Departamento de Física, Universidade Estadual Paulista, \\ 13506-970, Rio Claro, São Paulo, Brazil \\ ${ }^{2}$ Departamento de Física e Química, Universidade Estadual Paulista, 15385-000, Ilha Solteira, São Paulo, Brazil \\ ${ }^{3}$ Instituto de Física, Universidade Federal de Uberlândia, 38400-902, Uberlândia, Minas Gerais, Brazil \\ ${ }^{4}$ Division of Physics and Applied Physics, Nanyang Technological University, Singapore 637371, Singapore \\ ${ }^{5}$ Science Institute, University of Iceland, Dunhagi-3, IS-107, Reykjavik, Iceland
}

(Received 13 August 2013; revised manuscript received 18 October 2013; published 12 November 2013)

\begin{abstract}
We explore theoretically the density of states (LDOS) probed by a scanning tunneling microscope (STM) tip of two-dimensional systems hosting an adatom and a subsurface impurity, both capacitively coupled to atomic force microscope (AFM) tips and traversed by antiparallel magnetic fields. Two kinds of setups are analyzed, a monolayer of graphene and a two-dimensional electron gas (2DEG). The AFM tips set the impurity levels at the Fermi energy, where two contrasting behaviors emerge: The Fano factor for the graphene diverges, while in the 2DEG it approaches zero. As result, the spin degeneracy of the LDOS is lifted exclusively in the graphene system, in particular, for the asymmetric regime of Fano interference. The aftermath of this limit is a counterintuitive phenomenon, which consists of a dominant Fano factor due to the subsurface impurity even with a stronger STM-adatom coupling. Thus we find a full polarized conductance, achievable just by displacing vertically the position of the STM tip. Our work proposes the Fano effect as the mechanism to filter spins in graphene. This feature arises from the massless Dirac electrons within the band structure and allows us to employ the graphene host as a relativistic Fano spin filter.
\end{abstract}

DOI: 10.1103/PhysRevB.88.195122

PACS number(s): 07.79.Cz, 72.80.Vp, 05.60.Gg, 72.25.-b

\section{INTRODUCTION}

Graphene is a two-dimensional layer of atoms organized in a honeycomb lattice. Its peculiar band structure, consisting of two Dirac cones placed at the corners of the Brillouin zone and characterized by a massless relativistic dispersion relation, provides the opportunity for scientists to explore relativistic phenomena in the domain of condensed matter physics. In the past decade graphene was in focus of the physical community, both theoretically and experimentally. In particular, transport properties of graphene and other carbon-based nanostructures attracted the vivid interest of researchers. ${ }^{1-12}$

Recent experimental ${ }^{13,14}$ and theoretical ${ }^{15-18}$ studies reveal the possibility of the effective absorption of the individual magnetic impurities by single graphene sheets. The presence of such impurities (adatoms) strongly modifies the magnetic ${ }^{19-21}$ and transport properties of graphene ${ }^{22-24}$ which can be used for a variety of technological applications including chemical sensing. ${ }^{25,26}$ The convenient experimental techniques for the investigation of the properties of individual adatoms is provided by a scanning tunneling microscope (STM). ${ }^{27,28}$ The latter is made by a metallic tip that probes, for low enough temperatures, the local density of states (LDOS) of a sample by measuring the differential conductance. ${ }^{29,30}$ In this scenario, the STM of impurities adsorbed on graphene reveals the scattering of electrons in a relativistic environment.

The LDOS of metallic systems coupled to impurities displays Fano profiles ${ }^{31,32}$ resulting from the quantum interference between competing channels in the electron transport. Such an effect arises from the interplay between the paths of itinerant electrons that travel from the tip directly to the conduction band of the host and those that tunnel via impurity.
The total Fano factor, established by the superposition of these electron paths, defines the shape of the profiles of the differential conductance.

In the past decade particular attention has been devoted to the Fano effect in regular metals with magnetic adatoms in the Kondo limit. ${ }^{33}$ In this situation, the description of the host as a two-dimensional electron gas (2DEG) has been successfully verified. ${ }^{34-38}$

Additionally, in the emerging field of spintronics, the presence of spin-polarized hosts gives rise to interesting new features. ${ }^{39-55}$ For these cases, the splitting of the Kondo peak in the conductance characterizes the fingerprint of itinerant magnetism in the host. ${ }^{39,40}$ A spin-polarized tip and a nonmagnetic host also lead to a spin-dependent STM setup. Particularly, the aforementioned system behaves either as a spin filter in the Kondo regime or as a spin diode away from it. ${ }^{41-43}$ Moreover, a Fano spin filter can in principle be realized even in the absence of ferromagnetism ${ }^{56,57}$ and the Kondo effect. This can be achieved in the side-coupled geometry of a quantum wire hybridized with a quantum dot (QD) where spin degeneracy is lifted by the external magnetic field applied at the QD region. ${ }^{58}$

The properties of individual magnetic adatoms hosted by graphene were previously investigated theoretically by using the single-impurity Anderson Hamiltonian, ${ }^{59}$ both in the regime of high temperatures $T \gg T_{K}$ (the Kondo temperature) when the Hartree-Fock approach can be used ${ }^{5,6}$ and for $T \ll T_{K}$ when Kondo correlations become important. In the latter case, by changing the adatom level in the vicinity of the Fermi energy, it has been predicted that the Kondo peak arises in a narrower energy range than in normal metals. ${ }^{7}$ This is due to the difference in the dispersion of the carriers in 
(a)

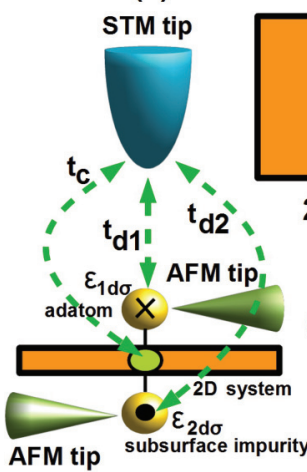

(b)

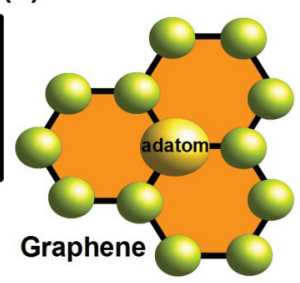

(c)



FIG. 1. (Color online) (a) A schematic diagram of the system considered in this paper. An STM tip is coupled to the atom adsorbed (adatom) in a 2D host which may be a $2 \mathrm{D}$ electron gas (2DEG) or a graphene sheet. A subsurface impurity is also considered which lies beneath the 2D system. The AFM tips allow us to control the energy levels of the impurities which are under the presence of antiparallel magnetic fields. The cross and dot at each impurity indicate the orientation of the magnetic field. (b) The top views of the setups considered: a $2 \mathrm{DEG}$ and a graphene sheet, respectively. (c) The Zeeman splitting caused by the magnetic field in both impurities.

the two systems: While the 2DEG is described by a parabolic dispersion, the honeycomb lattice of graphene leads to a linear dispersion relation near the Fermi level.

In the current paper we compare further the manifestations of spin-related phenomena in normal metals and graphene in the geometry of the two side-coupled impurities traversed by antiparallel magnetic fields [Figs. 1(a) and 1(b)]. These fields introduce a Zeeman splitting of the impurity levels as depicted in Fig. 1(c). Additionally, two atomic force microscope (AFM) tips are capacitively coupled to the impurities in order to set their energies at the host Fermi energy. ${ }^{8}$ These tips play the role of metallic gates usually employed to tune the levels of QDs embedded in nanostructures. ${ }^{60,61}$

The quantum interference between the alternative paths taken by the electrons rules the transport through the system and leads to the typical Fano shape of the profiles of the differential conductance of the system. They can be characterized in terms of Fano parameters which allow us to determine the relative impact of each path into the global response probed by the STM tip. The Fano parameters are dependent on the properties of the host and demonstrate opposite behavior in graphene and normal metals. In the former case, in the vicinity of the host Fermi energy, the Fano factor diverges, while in the latter case it approaches to zero. In order to explore such contrasting features, in our further consideration we set the levels of the impurities at the Fermi energy. As it will be shown in this paper, the lifting of the spin degeneracy of the LDOS is only feasible for the graphene system, in particular, in the asymmetric limit of Fano parameters. In this regime, a counterintuitive phenomenon is revealed, which is due to the Fano factor of the subsurface impurity that dominates the interference even with a stronger STM-adatom coupling. We find that the majority spin component of the LDOS can be tuned by displacing vertically the STM tip towards (or away from) the host. We also demonstrate that there is an STM tip position where the conductance becomes full polarized. The graphene host thus allows us to emulate an ideal relativistic Fano spin filter on massless Dirac fermions.

In order to model the system illustrated in Fig. 1, an approach based on the two-impurity Anderson Hamiltonian and going beyond the Hartree-Fock approximation and valid away from the Kondo regime was developed. We consider both cases of $2 \mathrm{DEG}$ and a graphene monolayer. In the latter system, we follow the approach proposed in Ref. 7, where an impurity is adsorbed above a single site of the host. Additionally, we take into account a subsurface impurity, situated opposite to the adatom. By using an equation-of-motion technique for the Green's functions we derive a spin-resolved formula for the LDOS, characterized by Fano interference parameters.

The paper is organized as follows. In Sec. II we present the theoretical model of metallic surfaces with two impurities and derive the expression for spin-dependent LDOS for the setups shown in Fig. 1. The decoupling scheme Hubbard $\mathrm{I}^{62}$ for the calculation of the Green's functions is presented in Sec. III. In Sec. IV we derive the expressions of the noninteracting self-energies of the impurities as well as the Fano parameters for the graphene sheet and the 2DEG, respectively. The results of the calculations are presented and discussed in Sec. V. Conclusions are summarized in Sec. VI.

\section{THEORETICAL MODEL}

\section{A. Hamiltonian}

In order to probe the LDOS of metallic surfaces, we consider an STM tip weakly connected to hosts hybridized with a pair of side-coupled impurities as outlined in Fig. 1. The systems we investigate are described according to the Hamiltonian

$$
\mathcal{H}=\mathcal{H}^{2 \mathrm{D}}+\mathcal{H}_{\text {tip }}+\mathcal{H}_{\text {tun }}
$$

The first term represents the Anderson model ${ }^{7}$ given by

$$
\begin{aligned}
\mathcal{H}^{2 \mathrm{D}}= & \sum_{s \sigma} \int d k \varepsilon_{k} c_{s k \sigma}^{\dagger} c_{s k \sigma}+\sum_{j \sigma} \varepsilon_{j d \sigma} d_{j \sigma}^{\dagger} d_{j \sigma} \\
& +\sum_{j} \mathcal{U}_{j} n_{j \uparrow} n_{j \downarrow}+\sum_{j s \sigma} \int d k \mathcal{V}_{j k}\left(c_{s k \sigma}^{\dagger} d_{j \sigma}+\text { H.c. }\right) .
\end{aligned}
$$

The surface electrons forming the hosts are described by the operators $c_{s k \sigma}^{\dagger}\left(c_{s k \sigma}\right)$ for the creation (annihilation) of an electron in a quantum state labeled by the wave number $k$, spin $\sigma$, and in the case of the graphene, an additional index $s$ standing for the valley index. ${ }^{4}$ For the $2 \mathrm{DEG}$, the quantum number $s$ does not exist. The dispersion relation for the graphene electrons is

$$
\varepsilon_{k}=\hbar v_{F} k
$$

with $\hbar$ as the Planck constant divided by $2 \pi$ and $v_{F}$ as the Fermi velocity. For the impurities, $d_{j \sigma}^{\dagger}\left(d_{j \sigma}\right)$ creates (annihilates) an electron with spin $\sigma$ in the state $\varepsilon_{j d \sigma}$, with the index $j=1,2$ corresponding to the upper and lower impurities. 
The magnetic fields split the energies $\varepsilon_{j d \sigma}$,

$$
\varepsilon_{j d \sigma}=\varepsilon_{j d}-\sigma \frac{\Delta_{j}}{2},
$$

where $\Delta_{j}$ is the Zeeman energy. Here we employ antiparallel magnetic fields established by the condition $\Delta_{1}=-\Delta_{2}$.

The third term in Eq. (2) accounts for the on-site Coulomb interaction $U_{j}$, with $n_{j \sigma}=d_{j \sigma}^{\dagger} d_{j \sigma}$. Finally, the last two terms mix the host continuum of states of the conduction band and the levels $\varepsilon_{j d \sigma}$, where H.c. stands for the Hermitian conjugate of the first term. This hybridization occurs at the impurity sites via the coupling

$$
\mathcal{V}_{j k}=\frac{v_{0}}{2 \pi} \sqrt{\frac{\pi \Omega_{0}}{\mathcal{N}}} \sqrt{|k|},
$$

where $\mathcal{N}$ is the number of conduction states, and the parameters $v_{0}$ and $\Omega_{0}$ denote the host-impurity hybridization in energy dimensions and the unit cell area, respectively.

The densities of states of the hosts per spin are different for graphene and 2DEG and are given by the expressions

$$
\rho_{0}=\rho^{\mathrm{GS}}(\varepsilon)=\sum_{s} \frac{\Omega_{0}}{2 \pi} \frac{|\varepsilon|}{\left(\hbar v_{F}\right)^{2}}=\frac{|\varepsilon|}{D^{2}}
$$

and

$$
\rho_{0}=\rho^{2 \mathrm{DEG}}(\varepsilon)=\frac{\Omega_{0}}{2 \pi} \frac{D}{\left(\hbar v_{F}\right)^{2}}=\frac{1}{2 D},
$$

where $2 D$ denotes the band width.

The second part of Eq. (1) is described by the Hamiltonian

$$
\mathcal{H}_{\text {tip }}=\sum_{\vec{q} \sigma} \varepsilon_{\vec{q}} b_{\vec{q} \sigma}^{\dagger} b_{\vec{q} \sigma}
$$

which corresponds to free electrons in the STM tip with energy $\varepsilon_{\vec{q}}$ ruled by fermionic operators $b_{\vec{q} \sigma}^{\dagger}$ and $b_{\vec{q} \sigma}$.

To perform the coupling between Eqs. (2) and (8), we have to define the tunneling Hamiltonian, here expressed in two distinct forms,

$$
\begin{aligned}
\mathcal{H}_{\mathrm{tun}} & =t_{c} \sum_{\sigma} \psi_{\mathrm{tip}, \sigma}^{\dagger} \psi_{\mathrm{host}, \sigma}+\sum_{j \sigma} t_{d j} \psi_{\mathrm{tip}, \sigma}^{\dagger} d_{j \sigma}+\text { H.c. } \\
& =t_{c} \sum_{\sigma} \psi_{\mathrm{tip}, \sigma}^{\dagger} \psi_{\sigma}+\text { H.c. }
\end{aligned}
$$

where

$$
\psi_{\text {tip }, \sigma}=\frac{1}{\sqrt{\mathcal{N}}} \sum_{\vec{q}} b_{\vec{q} \sigma}
$$

is the fermionic operator for the edge site of the tip,

$$
\psi_{\text {host }, \sigma}=\frac{1}{2 \pi} \sqrt{\frac{\pi \Omega_{0}}{\mathcal{N}}} \sum_{s} \int \sqrt{|k|} d k c_{s k \sigma}
$$

for the site of the host side-coupled to the impurities and

$$
\psi_{\sigma}=\frac{1}{2 \pi} \sqrt{\frac{\pi \Omega_{0}}{\mathcal{N}}} \sum_{s} \int \sqrt{|k|} d k c_{s k \sigma}+\left(\pi \rho_{0} v_{0}\right) \sum_{j} q_{j}^{e} d_{j \sigma}
$$

represents the operator that accounts Fano interference via the extrinsic Fano factor defined by

$$
q_{j}^{e}=\left(\pi \rho_{0} v_{0}\right)^{-1}\left(\frac{t_{d j}}{t_{c}}\right) .
$$

Notice that the electrons from the tip are able to tunnel directly to three different sites with different hopping terms denoted as: tunneling tip-host $\left(t_{c}\right)$, tunneling tip-adatom $\left(t_{d 1}\right)$ and tipsubsurface impurity $\left(t_{d 2}\right)$. It is worth mentioning that according to Refs. 63 and 64, these amplitudes are overlap integrals that mixture the STM tip with the "host + impurities" system. Thus by changing such parameters, we mimic the vertical displacement of the STM tip.

\section{B. LDOS for the "host + impurities" system in the presence of STM and AFM tips}

By applying the linear response theory, in which the STM tip is considered as a probe, it is possible to show that the differential conductance per spin is determined by ${ }^{6}$

$$
\mathcal{G}^{\sigma}(V) \sim \frac{e^{2}}{h} \pi \Gamma_{c} \rho_{\mathrm{LDOS}}^{\sigma}(e V),
$$

where $e$ is the electron charge, $\Gamma_{c}=4 \pi t_{c}^{2} \rho_{\text {tip }}, t_{c}$ is the tunneling term between the STM tip and the host, $\rho_{\text {tip }}$ is the DOS for the tip, $V$ is the bias voltage, and $\rho_{\mathrm{LDOS}}^{\sigma}$ is the spin-resolved LDOS of the "host + impurities" system.

To obtain the LDOS, we introduce for the operator of Eq. (12), the retarded Green's function

$$
\mathcal{R}_{\sigma}(t)=-\frac{i}{\hbar} \theta(t) \operatorname{Tr}\left\{\varrho_{2 \mathrm{D}}\left[\psi_{\sigma}(t), \psi_{\sigma}^{\dagger}(0)\right]_{+}\right\}
$$

in the time domain, where $\theta(t)$ is the Heaviside function, $\varrho_{2 \mathrm{D}}$ is the density matrix of the system described by the Hamiltonian [Eq. (2)], and $[\ldots, \ldots]_{+}$is the anticommutator of the field operator taken in Heisenberg picture. ${ }^{39}$

From Eq. (15), the spin-resolved LDOS of the host can be obtained as

$$
\rho_{\mathrm{LDOS}}^{\sigma}=-\frac{1}{\pi} \operatorname{Im}\left(\tilde{\mathcal{R}}_{\sigma}\right),
$$

where $\tilde{\mathcal{R}}_{\sigma}$ is the Fourier transform of $\mathcal{R}_{\sigma}(t)$. To obtain an analytical expression for the LDOS, we apply the equation-ofmotion approach to Eq. (15). Substituting Eq. (12) in Eq. (15), one gets

$$
\begin{aligned}
\mathcal{R}_{\sigma}(t)= & \left(\frac{1}{2 \pi} \sqrt{\frac{\pi \Omega_{0}}{\mathcal{N}}}\right)^{2} \sum_{s \tilde{s}} \int \sqrt{|k|} d k \sqrt{|q|} d q \mathcal{R}_{c_{s k} c_{\tilde{s} q}}^{\sigma} \\
& +\left(\pi \rho_{0} v_{0}\right) \sum_{j s} q_{j}^{e}\left(\frac{1}{2 \pi} \sqrt{\frac{\pi \Omega_{0}}{\mathcal{N}}}\right) \int \sqrt{|k|} d k \\
& \times\left(\mathcal{R}_{d_{j} c_{s k}}^{\sigma}+\mathcal{R}_{c_{s k} d_{j}}^{\sigma}\right)+\left(\pi \rho_{0} v_{0}\right)^{2} \sum_{j l} q_{j}^{e} q_{l}^{e} \mathcal{R}_{d_{j} d_{l}}^{\sigma},
\end{aligned}
$$

expressed in terms of the Green's functions $\mathcal{R}_{c_{s k} c_{s q}}^{\sigma}, \mathcal{R}_{d_{j} c_{s k}}^{\sigma}$, $\mathcal{R}_{c_{s k} d_{j}}^{\sigma}$, and $\mathcal{R}_{d_{j} d_{l}}^{\sigma}$.

First, we have to determine

$$
\mathcal{R}_{c_{s k} c_{\tilde{s} q}}^{\sigma}(t)=-\frac{i}{\hbar} \theta(t) \operatorname{Tr}\left\{\varrho_{2 \mathrm{D}}\left[c_{s k \sigma}(t), c_{\tilde{s} q \sigma}^{\dagger}(0)\right]_{+}\right\}
$$


by acting by the operator $\partial_{t} \equiv \frac{\partial}{\partial t}$ on Eq. (18). We find

$$
\begin{aligned}
\partial_{t} \mathcal{R}_{c_{s k} c_{\tilde{s} q}}^{\sigma}(t)= & -\frac{i}{\hbar} \delta(t) \operatorname{Tr}\left\{\varrho_{2 \mathrm{D}}\left[c_{s k \sigma}(t), c_{\tilde{s} q \sigma}^{\dagger}(0)\right]_{+}\right\} \\
& -\frac{i}{\hbar} \varepsilon_{k} \mathcal{R}_{c_{s k} c_{\tilde{s} q}}^{\sigma}(t)-\frac{i}{\hbar} \sum_{j} \mathcal{V}_{j k} \mathcal{R}_{d_{j} c_{\tilde{s} q}}^{\sigma}(t),
\end{aligned}
$$

where we have used

$$
i \hbar \partial_{t} c_{s k \sigma}(t)=\left[c_{s k \sigma}, \mathcal{H}^{2 \mathrm{D}}\right]=\varepsilon_{k} c_{s k \sigma}(t)+\sum_{j} \mathcal{V}_{j k} d_{j \sigma}(t)
$$

In the energy domain, we solve Eq. (19) for $\tilde{\mathcal{R}}_{c_{s k} c_{\tilde{s q}}}^{\sigma}$ and obtain

$$
\tilde{\mathcal{R}}_{c_{s k} c_{\tilde{s} q}}^{\sigma}=\frac{\delta(k-q) \delta_{s \tilde{s}}}{\varepsilon^{+}-\varepsilon_{k}}+\sum_{j} \frac{\mathcal{V}_{j k}}{\varepsilon^{+}-\varepsilon_{k}} \tilde{\mathcal{R}}_{d_{j} c_{s q}}^{\sigma},
$$

where $\varepsilon^{+}=\varepsilon+i \eta$ and $\eta \rightarrow 0^{+}$. Notice that we also need to calculate the mixed Green's function $\tilde{\mathcal{R}}_{d_{j} c_{s q}}^{\sigma}$. To this end, we define the advanced Green's function

$$
\mathcal{A}_{d_{j} c_{s q}}^{\sigma}(t)=\frac{i}{\hbar} \theta(-t) \operatorname{Tr}\left\{\varrho_{2 \mathrm{D}}\left[d_{j \sigma}^{\dagger}(0), c_{s q \sigma}(t)\right]_{+}\right\},
$$

whose equation of motion reads

$$
\begin{aligned}
\partial_{t} \mathcal{A}_{d_{j} c_{s q}}^{\sigma}(t)= & -\frac{i}{\hbar} \delta(t) \operatorname{Tr}\left\{\varrho_{2 \mathrm{D}}\left[d_{j \sigma}^{\dagger}(0), c_{s q \sigma}(t)\right]_{+}\right\} \\
& -\frac{i}{\hbar} \varepsilon_{q} \mathcal{A}_{d_{j} c_{s q}}^{\sigma}(t)-\frac{i}{\hbar} \sum_{l} \mathcal{V}_{l q} \mathcal{A}_{d_{j} d_{l}}^{\sigma}(t),
\end{aligned}
$$

where we have used once again Eq. (20), interchanging $k \leftrightarrow q$. The Fourier transform of Eq. (23) leads to

$$
\varepsilon^{-} \tilde{\mathcal{A}}_{d_{j} c_{s q}}^{\sigma}=\varepsilon_{q} \tilde{\mathcal{A}}_{d_{j} c_{s q}}^{\sigma}+\sum_{l} \mathcal{V}_{l q} \tilde{\mathcal{A}}_{d_{j} d_{l}}^{\sigma},
$$

with $\varepsilon^{-}=\varepsilon-i \eta$. Applying the property $\tilde{\mathcal{R}}_{d_{j} c_{s q}}^{\sigma}=\left(\tilde{\mathcal{A}}_{d_{j} c_{s q}}^{\sigma}\right)^{\dagger}$ on Eq. (24), we show that

$$
\begin{aligned}
\varepsilon^{+} \tilde{\mathcal{R}}_{d_{j} c_{s q}}^{\sigma} & =\varepsilon_{q} \tilde{\mathcal{R}}_{d_{j} c_{s q}}^{\sigma}+\sum_{l} \mathcal{V}_{l q} \tilde{\mathcal{R}}_{d_{j} d_{l}}^{\sigma}, \\
\tilde{\mathcal{R}}_{d_{j} c_{s q}}^{\sigma} & =\sum_{l} \frac{\mathcal{V}_{l q}}{\varepsilon^{+}-\varepsilon_{q}} \tilde{\mathcal{R}}_{d_{j} d_{l}}^{\sigma},
\end{aligned}
$$

and analogously,

$$
\tilde{\mathcal{R}}_{c_{s q} d_{j}}^{\sigma}=\sum_{l} \frac{\mathcal{V}_{l q}}{\varepsilon^{+}-\varepsilon_{q}} \tilde{\mathcal{R}}_{d_{l} d_{j}}^{\sigma} .
$$

Now we substitute Eq. (26) into Eq. (21) and the latter, together with Eq. (27), into Eq. (17) and determine

$$
\begin{aligned}
\tilde{\mathcal{R}}_{\sigma}= & \left(\frac{1}{2 \pi} \sqrt{\frac{\pi \Omega_{0}}{\mathcal{N}}}\right)^{2} \sum_{s} \int k d k \frac{1}{\varepsilon^{+}-\varepsilon_{k}} \\
& +\left(\pi \rho_{0} v_{0}\right)^{2} \sum_{j l}\left(q_{j}-i \mathcal{F}_{j}\right) \tilde{\mathcal{R}}_{d_{j} d_{l}}^{\sigma}\left(q_{l}-i \mathcal{F}_{l}\right) \\
& +\left(\pi \rho_{0} v_{0}\right)^{2} \sum_{j l} q_{j}^{e}\left(q_{l}-i \mathcal{F}_{l}\right)\left(\tilde{\mathcal{R}}_{d_{j} d_{l}}^{\sigma}+\tilde{\mathcal{R}}_{d_{l} d_{j}}^{\sigma}\right) \\
& +\left(\pi \rho_{0} v_{0}\right)^{2} \sum_{j l} q_{j}^{e} q_{l}^{e} \tilde{\mathcal{R}}_{d_{j} d_{l}}^{\sigma},
\end{aligned}
$$

where

$$
q_{j}=\frac{1}{\pi \rho_{0} v_{0}^{2}} \operatorname{Re} \Sigma_{j j}
$$

is the Fano parameter ${ }^{6}$ due to the host-impurity coupling and

$$
\mathcal{F}_{j}=-\frac{1}{\pi \rho_{0} v_{0}^{2}} \operatorname{Im} \Sigma_{j j},
$$

with

$$
\Sigma_{l \tilde{l}}=\sum_{s} \int d k \frac{\mathcal{V}_{l k} \mathcal{V}_{\tilde{l} k}}{\varepsilon^{+}-\varepsilon_{k}}
$$

being the noninteracting self-energy of the impurities. ${ }^{6}$ From Eqs. (16) and (28), we finally derive the spin-resolved LDOS

$$
\rho_{\mathrm{LDOS}}^{\sigma}=\rho_{1122}^{\sigma}+\rho_{1221}^{\sigma},
$$

where

$$
\begin{aligned}
\rho_{1122}^{\sigma}= & \rho_{0}+\rho_{0} \Gamma \sum_{j}\left[\left(\mathcal{F}_{j}^{2}-q_{T j}^{2}\right) \operatorname{Im}\left(\tilde{\mathcal{R}}_{d_{j} d_{j}}^{\sigma}\right)\right. \\
& \left.+2 q_{T j} \mathcal{F}_{j} \operatorname{Re}\left(\tilde{\mathcal{R}}_{d_{j} d_{j}}^{\sigma}\right)\right]
\end{aligned}
$$

is the direct term of the LDOS and

$$
\begin{aligned}
\rho_{1221}^{\sigma}= & \rho_{0} \Gamma \sum_{j \neq l}\left[\left(\mathcal{F}_{j} \mathcal{F}_{l}-q_{T j} q_{T l}\right) \operatorname{Im}\left(\tilde{\mathcal{R}}_{d_{j} d_{l}}^{\sigma}\right)\right. \\
& \left.+\left(q_{T j} \mathcal{F}_{l}+q_{T l} \mathcal{F}_{j}\right) \operatorname{Re}\left(\tilde{\mathcal{R}}_{d_{j} d_{l}}^{\sigma}\right)\right]
\end{aligned}
$$

represents the mixing term that arises from the interference between the impurities, with

$$
q_{T j}=q_{j}+q_{j}^{e}
$$

being the total Fano factor and $\Gamma=\pi v_{0}^{2} \rho_{0}$ is the Anderson parameter.

Equation (32) is the main analytical result of this paper. It describes the spin-resolved LDOS of 2D systems with two impurities in the side-coupled geometry shown in Fig. 1. This equation shows the dependence of the LDOS on the direct and mixed Green's functions of the impurities $\tilde{\mathcal{R}}_{d_{j} d_{j}}^{\sigma}$ and $\tilde{\mathcal{R}}_{d_{j} d_{l}}^{\sigma}$, respectively, and on the total Fano parameter given by Eq. (35). We highlight that the Zeeman energy of the impurities determines the spin dependence of the LDOS and, therefore, the spin-filter behavior, in particular, only for the graphene system, as we will see.

In order to investigate the spin dependence of the LDOS as well as the spin-filter effect, we introduce the expression

$$
\operatorname{LDOS}=\frac{\rho_{\mathrm{LDOS}}^{\uparrow}+\rho_{\mathrm{LDOS}}^{\downarrow}}{\rho^{\mathrm{GS}}(D)}
$$

for the dimensionless LDOS, where we have used Eq. (6) for $\rho^{\mathrm{GS}}(D)$ and

$$
\mathrm{SP}=\frac{\mathcal{G}^{\uparrow}-\mathcal{G}^{\downarrow}}{\mathcal{G}^{\uparrow}+\mathcal{G}^{\downarrow}}
$$

for the transport polarization of the system settled from Eq. (14). 


\section{GREEN'S FUNCTIONS OF THE IMPURITIES}

In the present section we calculate $\tilde{\mathcal{R}}_{d_{j} d_{l}}^{\sigma}(j, l=1,2)$ within the Hubbard I approximation. ${ }^{62}$ This approach provides reliable results away from the Kondo regime. Thus we begin by applying the equation-of-motion method on these Green's functions, which results in

$\left(\varepsilon^{+}-\varepsilon_{l d \sigma}\right) \tilde{\mathcal{R}}_{d_{l} d_{j}}^{\sigma}=\delta_{l j}+\sum_{\tilde{l}} \Sigma_{l \tilde{l}} \tilde{\mathcal{R}}_{d_{l} d_{j}}^{\sigma}+\mathcal{U}_{l} \tilde{\mathcal{R}}_{d_{l \sigma} n_{d_{l} \bar{\sigma}}, d_{j \sigma}}$.

In the equation above, $\tilde{\mathcal{R}}_{d_{l \sigma} n_{d_{l} \bar{\sigma}}, d_{j \sigma}}$ is a two-particle Green's function composed of four fermionic operators, obtained from the time Fourier transform of

$$
\mathcal{R}_{d_{l \sigma} n_{d_{l} \bar{\sigma}}, d_{j \sigma}}=-\frac{i}{\hbar} \theta(t) \operatorname{Tr}\left\{\varrho_{2 \mathrm{D}}\left[d_{l \sigma}(t) n_{d_{l} \bar{\sigma}}(t), d_{j \sigma}^{\dagger}(0)\right]_{+}\right\},
$$

with $n_{d_{l} \bar{\sigma}}=d_{l \bar{\sigma}}^{\dagger} d_{l \bar{\sigma}}$ and spin $\bar{\sigma}$ (opposite to $\sigma$ ).

In order to close the system of Green's functions in Eq. (38), we calculate the time derivative of Eq. (39) and then its time Fourier transform, which leads to

$$
\begin{aligned}
\left(\varepsilon^{+}\right. & \left.-\varepsilon_{l d \sigma}-\mathcal{U}_{l}\right) \tilde{\mathcal{R}}_{d_{l \sigma} n_{d \bar{\sigma}}, d_{j \sigma}} \\
= & \delta_{l j}\left\langle n_{d_{l} \bar{\sigma}}\right\rangle+\sum_{s} \int d k \mathcal{V}_{l k}\left(\tilde{\mathcal{R}}_{c_{s k \sigma} d_{l \bar{\sigma}}^{\dagger} d_{l \bar{\sigma}}, d_{j \sigma}}\right. \\
& \left.\quad-\tilde{\mathcal{R}}_{c_{s k \bar{\sigma}}^{\dagger} d_{l \bar{\sigma}} d_{l \sigma}, d_{j \sigma}}+\tilde{\mathcal{R}}_{d_{l \bar{\sigma}}^{\dagger} c_{s k \bar{\sigma}} d_{l \sigma}, d_{j \sigma}}\right),
\end{aligned}
$$

expressed in terms of new Green's functions of the same order of $\tilde{\mathcal{R}}_{d_{l \sigma} n_{d_{l} \bar{\sigma}}, d_{j \sigma}}$ and the occupation number

$$
\left\langle n_{d_{l} \bar{\sigma}}\right\rangle=-\frac{1}{\pi} \int_{-D}^{\epsilon_{F}=0} \operatorname{Im}\left(\tilde{\mathcal{R}}_{d_{l} d_{l}}^{\bar{\sigma}}\right) d \varepsilon,
$$

determined in accordance with Refs. 5 and 6. By employing the Hubbard I approximation, we decouple the Green's functions in the right-hand side of Eq. (40) as follows: $\tilde{\mathcal{R}}_{c_{s k \bar{\sigma}}^{\dagger} d_{l \bar{\sigma}} d_{l \sigma}, d_{j \sigma}} \simeq$ $\left\langle c_{s k \bar{\sigma}}^{\dagger} d_{l \bar{\sigma}}\right\rangle \tilde{\mathcal{R}}_{d_{l} d_{j}}^{\sigma}$ and $\tilde{\mathcal{R}}_{d_{l \bar{\sigma}}^{\dagger} c_{s k \bar{\sigma}} d_{l \sigma}, d_{j \sigma}} \simeq\left\langle c_{s k \bar{\sigma}}^{\dagger} d_{l \bar{\sigma}}\right\rangle \tilde{\mathcal{R}}_{d_{l} d_{j}}^{\sigma}$. As a result, we find

$$
\begin{aligned}
& \left(\varepsilon^{+}-\varepsilon_{l d \sigma}-\mathcal{U}_{l}\right) \tilde{\mathcal{R}}_{d_{l \sigma} n_{d_{l} \bar{\sigma}}, d_{j \sigma}} \\
& \quad=\delta_{l j}\left\langle n_{d_{l} \bar{\sigma}}\right\rangle+\left\langle n_{d_{l} \bar{\sigma}}\right\rangle\left(\sum_{s} \int d k \mathcal{V}_{l k}\right) \times \tilde{\mathcal{R}}_{c_{s k \sigma} d_{l \bar{\sigma}}^{\dagger} d_{l \bar{\sigma}}, d_{j \sigma}} .
\end{aligned}
$$

To close the calculation, we need to determine $\tilde{\mathcal{R}}_{c_{s k \sigma}} d_{l \bar{\sigma}}^{\dagger} d_{l \bar{\sigma}}, d_{j \sigma}$. Once again, employing the equation-of-motion approach for $\tilde{\mathcal{R}}_{c_{s k \sigma} d_{l \bar{\sigma}}^{\dagger} d_{l \bar{\sigma}}, d_{j \sigma}}$, we find

$$
\begin{aligned}
& \left(\varepsilon^{+}-\varepsilon_{k}\right) \tilde{\mathcal{R}}_{c_{s k \sigma} d_{l \bar{\sigma}}^{\dagger} d_{l \bar{\sigma}}, d_{j \sigma}} \\
& =\mathcal{V}_{l k} \tilde{\mathcal{R}}_{d_{l \sigma} n_{d_{l} \bar{\sigma}}, d_{j \sigma}}+\sum_{\tilde{s}} \int d q \mathcal{V}_{l q} \tilde{\mathcal{R}}_{c_{s k \sigma} d_{l \bar{\sigma}}^{\dagger} c_{\tilde{s} q \bar{\sigma}}, d_{j \sigma}} \\
& \quad+\sum_{\tilde{j} \neq l} \mathcal{V}_{\tilde{j} k} \tilde{\mathcal{R}}_{d_{\tilde{j} \sigma} n_{d_{l} \bar{\sigma}}, d_{j \sigma}}-\sum_{\tilde{s}} \int d q \mathcal{V}_{l q} \tilde{\mathcal{R}}_{c_{\tilde{s} q \bar{\sigma}}^{\dagger} d_{l \bar{\sigma}} c_{s k \sigma}, d_{j \sigma}} .
\end{aligned}
$$

For the sake of simplicity, we take the limit $\mathcal{U}_{l} \rightarrow$ $\infty$ and continue with the Hubbard I scheme by making $\tilde{\mathcal{R}}_{c_{s k \sigma} d_{l \bar{\sigma}}^{\dagger} c_{\tilde{s} q \bar{\sigma}}, d_{j \sigma}} \simeq\left\langle d_{l \bar{\sigma}}^{\dagger} c_{\tilde{s} q \bar{\sigma}}\right\rangle \tilde{\mathcal{R}}_{c_{s k \sigma} d_{j \sigma}}, \tilde{\mathcal{R}}_{c_{\tilde{s} q \bar{\sigma}}^{\dagger} d_{l \bar{\sigma}} c_{s k \sigma}, d_{j \sigma}} \simeq$ $\left\langle d_{l \bar{\sigma}}^{\dagger} c_{\tilde{s} q \bar{\sigma}}\right\rangle \tilde{\mathcal{R}}_{c_{s k \sigma} d_{j \sigma}}$, and $\tilde{\mathcal{R}}_{d_{\tilde{j} \sigma} n_{d_{l} \bar{\sigma}}, d_{j \sigma}} \simeq\left\langle n_{d_{l} \bar{\sigma}}\right\rangle \tilde{\mathcal{R}}_{d_{\tilde{j}} d_{j}}^{\sigma}$ in Eq. (43), which becomes

$$
\begin{aligned}
& \tilde{\mathcal{R}}_{c_{s k \sigma} d_{l \bar{\sigma}}^{\dagger} d_{l \bar{\sigma}}, d_{j \sigma}} \\
& \quad=\frac{\mathcal{V}_{l k}}{\left(\varepsilon^{+}-\varepsilon_{k}\right)} \tilde{\mathcal{R}}_{d_{l \sigma} n_{d_{l} \bar{\sigma}}, d_{j \sigma}}+\frac{\sum_{\tilde{j} \neq l} \mathcal{V}_{\tilde{j} k}}{\left(\varepsilon^{+}-\varepsilon_{k}\right)}\left\langle n_{d_{l} \bar{\sigma}}\right\rangle \tilde{\mathcal{R}}_{d_{j} d_{j}}^{\sigma} .
\end{aligned}
$$

Thus by solving the system of Green's functions composed by Eqs. (38), (42), (43), and (44), we obtain

$$
\tilde{\mathcal{R}}_{d_{1} d_{1}}^{\sigma}=\frac{1-\left\langle n_{d_{1} \bar{\sigma}}\right\rangle}{\varepsilon-\varepsilon_{1 d \sigma}-\sum_{11}-\lambda_{12}^{\bar{\sigma}} \frac{\left(\sum_{11}\right)^{2}}{\varepsilon-\varepsilon_{2 d \sigma}-\sum_{11}}},
$$

where $\lambda_{12}^{\bar{\sigma}}=\left(1-\left\langle n_{d_{1} \bar{\sigma}}\right\rangle\right)\left(1-\left\langle n_{d_{2} \bar{\sigma}}\right\rangle\right)$ and

$$
\tilde{\mathcal{R}}_{d_{2} d_{1}}^{\sigma}=\left(1-\left\langle n_{d_{2} \bar{\sigma}}\right\rangle\right) \frac{\sum_{21}}{\varepsilon-\varepsilon_{2 d \sigma}-\sum_{21}} \tilde{\mathcal{R}}_{d_{1} d_{1}}^{\sigma},
$$

with $\sum_{j l}$ determined by Eq. (31). We point out that the Green's functions $\tilde{\mathcal{R}}_{d_{2} d_{2}}^{\sigma}$ and $\tilde{\mathcal{R}}_{d_{1} d_{2}}^{\sigma}$ can be found by swapping $1 \leftrightarrow 2$ in Eqs. (45) and (46). As a result, Eq. (45) and $\tilde{\mathcal{R}}_{d_{2} d_{2}}^{\sigma}$ allow us to introduce

$$
\operatorname{DOS}_{j j}^{\sigma}=-\frac{1}{\pi \rho^{\mathrm{GS}}(D)} \operatorname{Im}\left(\tilde{\mathcal{R}}_{d_{j} d_{j}}^{\sigma}\right)
$$

as the dimensionless DOS for the impurities, where we have applied Eq. (6) at the band edge $D$.

\section{NONINTERACTING SELF-ENERGIES AND FANO PARAMETERS}

In this section we present the calculations of the noninteracting self-energies of Eq. (31) and the Fano parameters within Eqs. (13), (29), and (35). Equation (31) allows us to find

$$
\Sigma_{l \tilde{l}}=\Sigma_{l \tilde{l}}^{\mathrm{GS}}=\eta^{\mathrm{GS}}\left(\varepsilon \ln \left|\frac{\varepsilon^{2}}{\varepsilon^{2}-D^{2}}\right|-i \pi|\varepsilon|\right)
$$

for the graphene sheet, with

$$
\eta^{\mathrm{GS}}=\frac{\Omega_{0}}{2 \pi \mathcal{N}} \frac{v_{0}^{2}}{\left(\hbar v_{F}\right)^{2}}=\frac{v_{0}^{2}}{D^{2}}
$$

and

$$
\Sigma_{l \tilde{l}}=\Sigma_{l \tilde{l}}^{2 \mathrm{DEG}}=\eta^{2 \mathrm{DEG}}\left(D \ln \left|\frac{D+\varepsilon}{D-\varepsilon}\right|-i \pi D\right)
$$

for the case of the 2DEG, where

$$
\eta^{2 \mathrm{DEG}}=\frac{\Omega_{0}}{2 \pi \mathcal{N}} \frac{v_{0}^{2}}{\left(\hbar v_{F}\right)^{2}}=\frac{v_{0}^{2}}{2 D^{2}} .
$$

Notice that for $\varepsilon \ll D, \operatorname{Re}\left(\Sigma_{l \tilde{l}}^{\mathrm{GS}}\right)=\operatorname{Re}\left(\Sigma_{l \tilde{l}}^{2 \mathrm{DEG}}\right) \rightarrow 0$.

The self-energy of Eq. (48) is in accordance with the corresponding determined in Refs. 5-7. For the 2DEG, we recover the result found in Ref. 33.

From Eqs. (13), (29), (30), and (35), we determine

$$
q_{T j}^{\mathrm{GS}}=\frac{1}{\pi} \ln \left|\frac{\varepsilon^{2}}{\varepsilon^{2}-D^{2}}\right|+\left(\frac{t_{d j}}{t_{c}}\right) \frac{D^{2}}{\pi v_{0}} \frac{1}{|\varepsilon|}
$$

and

$$
\mathcal{F}_{j}^{\mathrm{GS}}=1
$$


for the graphene system, while for the 2DEG we have

$$
q_{T j}^{2 \mathrm{DEG}}=\frac{1}{\pi} \ln \left|\frac{D+\varepsilon}{D-\varepsilon}\right|+\left(\frac{t_{d j}}{t_{c}}\right) \frac{2 D}{\pi \nu_{0}}
$$

and

$$
\mathcal{F}_{j}^{2 \mathrm{DEG}}=1 .
$$

We emphasize that for $t_{d j} / t_{c} \ll 1$ and energies $\varepsilon \ll D$, Eqs. (52) and (54) exhibit opposite behaviors: $\left|q_{T j}^{\mathrm{GS}}\right| \rightarrow \infty$ and $\left|q_{T j}^{2 \mathrm{DEG}}\right| \rightarrow 0$.

\section{NUMERICAL RESULTS}

The present approach is for $T \gg T_{K}$ and within a range of temperatures where we can safely define the Heaviside step function as the Fermi distribution in the host. This assumption was previously considered in Refs. 5 and 6 . We measure the densities and energies in units of $\rho^{\mathrm{GS}}(D)$ and $\left[\rho^{\mathrm{GS}}(D)\right]^{-1}$, respectively, where we have used Eq. (6) at the band edge $D=7 \mathrm{eV}^{5,6}$ For the Zeeman splittings, we employ $\Delta_{1}=$ $-\Delta_{2}=2 \times 10^{-5}$ in Eq. (4) corresponding to a magnetic field of $\approx 2.4 \mathrm{~T}$. We have also used $v_{0}=0.14$ in Eq. (5). ${ }^{5,6}$ The levels of the impurities are set to $\varepsilon_{1 d}=\varepsilon_{2 d}=0$.

\section{A. Graphene system}

Here we analyze the graphene system by employing Eq. (36) in combination with Eqs. (6), (45), (46), (48), (52), and (53).

In Fig. 2, we present the absolute value of the Fano parameter $\left|q_{T j}^{\mathrm{GS}}\right|$ of Eq. (52) as a function of the energy $\varepsilon$. All curves exhibit a general trend in which the Fano factor tends to infinity as the energy approaches the Fermi level $(\varepsilon=0)$ and decays to a finite value as $\varepsilon$ increases towards the band edge. By increasing the ratio $t_{d j} / t_{c}$ the Fano parameter diverges more rapidly. In Fig. 2(a) the curve for $\left|q_{T 1}^{\mathrm{GS}}\right|$ (solid blue curve) becomes broader than the curve for $\left|q_{T 2}^{\mathrm{GS}}\right|$ (dotted red curve) for $|\varepsilon| \lesssim 0.2$ and keeps increasing as $\varepsilon \rightarrow 0$. This behavior becomes even more pronounced in Fig. 2(b) where the ratio $t_{d 1} / t_{c}$ is increased ten times while $t_{d 2} / t_{c}$ is kept fixed. As a result, $\left|q_{T 1}^{\mathrm{GS}}\right|$ lies above the $\left|q_{T 2}^{\mathrm{GS}}\right|$ for almost the entire range except in the borders of the scale for $|\varepsilon| \gtrsim 1.7$. The shaded regions in Fig. 2 are defined in such a way that $\left|q_{T 2}^{\mathrm{GS}}\right|<\left|q_{T 1}^{\mathrm{GS}}\right|$ while out of these regions the opposite relation is verified, i.e., $\left|q_{T 2}^{\mathrm{GS}}\right|>\left|q_{T 1}^{\mathrm{GS}}\right|$. In the last case, the resulting behavior yields a Fano interference in the LDOS dictated by the subsurface impurity, where $\left|q_{T 2}^{\mathrm{GS}}\right|$ becomes dominant. It is worth mentioning that in spite of the condition $t_{d 1} / t_{c}>t_{d 2} / t_{c}$ which is maintained for all curves in Fig. 2, there are regions of $\varepsilon$ in which the opposite condition $\left(\left|q_{T 2}^{\mathrm{GS}}\right|>\left|q_{T 1}^{\mathrm{GS}}\right|\right)$ is verified. This unexpected feature is a result of the interplay between the quantum interference in the double impurity system and the peculiar behavior of the graphene density of states. We point out that such a behavior is not present in the 2DEG setup, as we will verify in Sec. V B.

In Fig. 2(b), we move the STM tip closer to the host by choosing $t_{d 1} / t_{c}=10 \times 10^{-5}$. Within the shaded regions, the adatom gives the dominant impact to the interference and $\left|q_{T 1}^{\mathrm{GS}}\right|$ (solid blue curve) overcomes $\left|q_{T 2}^{\mathrm{GS}}\right|$ (dotted red curve). When $t_{d j} / t_{c} \ll 1$, the profile of the LDOS for the graphene
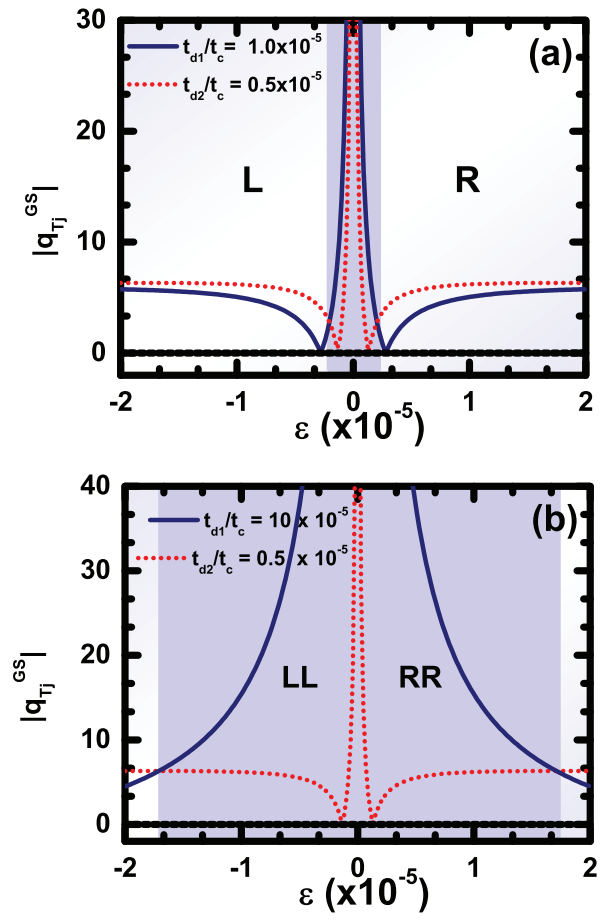

FIG. 2. (Color online) Absolute value of the Fano parameter of Eq. (52) as a function of the energy $\varepsilon$ for $v_{0}=0.14$ and $t_{d 2} / t_{c}=0.5 \times$ $10^{-5}$. (a) $t_{d 1} / t_{c}=1 \times 10^{-5}$. (b) $t_{d 1} / t_{c}=10 \times 10^{-5}$. In these graphs we have defined a shaded region in which the condition $\left|q_{T 2}^{\mathrm{GS}}\right|<\left|q_{T 1}^{\mathrm{GS}}\right|$ is verified. By increasing the ratio $t_{d 1} / t_{c}$ from $1 \times 10^{-5}$ to $10 \times 10^{-5}$, this region is enlarged as shown in (b). For both cases it is observed that the Fano factor tends to infinity as the energy approaches the Fermi level.

is expected to exhibit resonances. This result contrasts with the 2DEG system, where standard Fano's theory predicts antiresonances. In fact, Eq. (54) ensures $\left|q_{T j}^{2 \mathrm{DEG}}\right| \rightarrow 0$ for $\varepsilon \rightarrow 0 . .^{31,32}$

As it was mentioned earlier, the spin components of the DOSs for the impurities [Eq. (47)] are displaced in opposite directions away from the Fermi level $(\varepsilon=0)$ as Eq. (4) ensures. In Fig. 3(a), the Zeeman energy is $\Delta_{1}=2 \times 10^{-5}$, thus the resonance of the localized state in the adatom for spin up (dotted red curve) moves to the left, while the corresponding for spin down (solid blue curve) goes to the right (see the "down" and "up" drawn arrows in this figure). We identify such resonances by the letters "AA" and "BB." For the subsurface impurity, $\Delta_{2}=-\Delta_{1}$ and the displacements of the peaks become reversed as displayed in Fig. 3(b) (the "down" and "up" drawn arrows illustrate such a process). These peaks are labeled as "A" and "B." The Fano factors shown in Fig. 2 and the peaks "A", "B," "AA," and "BB" for the spin-dependent resonances will help us to perceive, in the asymmetric limit $t_{d 1} / t_{c} \neq t_{d 2} / t_{c}$, the reversal of the majority spin component in the LDOS.

As we have antiparallel magnetic fields settled by the constraint $\Delta_{1}=-\Delta_{2}=2 \times 10^{-5}$ for the Zeeman energies, the LDOS does not exhibit spin dependence in the case of the symmetric limit of Fano factors, determined by the ratios $t_{d 1} / t_{c}=t_{d 2} / t_{c}$. The symmetric limit of Fano factors can be achieved by assuming that the overlap integral $t_{d 2}$ between the 

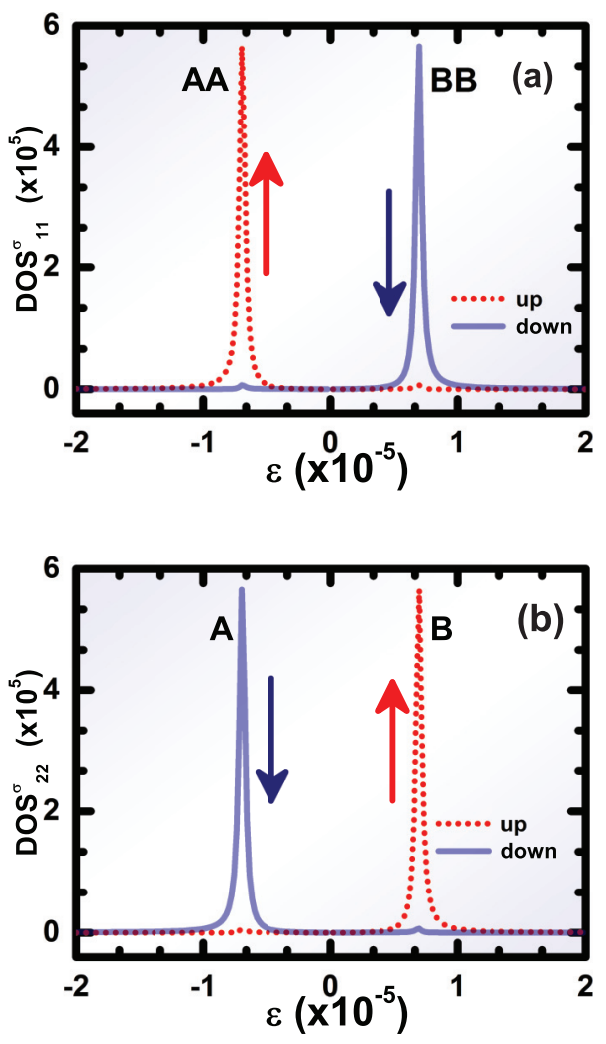

FIG. 3. (Color online) Parameters: $v_{0}=0.14$ and $\varepsilon_{1 d}=\varepsilon_{2 d}=0$. Here we use Eq. (47) for the DOS of the impurities. (a) DOS for the adatom with Zeeman energy $\Delta_{1}=2 \times 10^{-5}$. (b) DOS for the subsurface impurity with Zeeman energy $\Delta_{2}=-\Delta_{1}$ (antiparallel magnetic fields). The arrows (red and blue) indicate a given spin corresponding to the resonance.

tip and the subsurface impurity has a broader wave function that arises from the latter, which compensates the minor proximity of the STM tip, thus leading to the same overlap $t_{d 1}$ with the adatom.

Thus the profile of the LDOS is spin degenerate, as we can see in Fig. 4(a) for $t_{d 1} / t_{c}=t_{d 2} / t_{c}=1 \times 10^{-5}$, in which the curves for both spins are superimposed. Despite the unpolarized profile of the LDOS, we highlight that the pair of resonances found is a direct result of the Zeeman splittings at the sites of the impurities. Otherwise, for levels $\varepsilon_{1 d \sigma}=\varepsilon_{2 d \sigma}=0$, the host is completely decoupled from the impurities and the surface LDOS reduces to Eq. (6), whose form is illustrated by the green line with squares, also shown in Fig. 4(a).

In Fig. 4(b) we show the total LDOS (solid black curve) obtained from Eq. (36). It can be noted that both spin states contribute equally to the total LDOS for all values of energy $\varepsilon$. This is represented by two antiparallel arrows at both peaks for the total LDOS. Here we also make explicit the effect of the mixing term $\Sigma_{\sigma} \rho_{1221}^{\sigma}$ obtained from Eq. (34) upon the LDOS determined by Eq. (32). Figure 4(b) shows that the quantity $\Sigma_{\sigma} \rho_{1221}^{\sigma}$ (dotted gray curve) suppresses the direct term $\Sigma_{\sigma} \rho_{1122}^{\sigma}$ calculated from Eq. (33). The latter exhibits two resonances (dashed gray curve), while the former is characterized by two antiresonances. The peaks in the direct term are the hallmark of constructive interference, contrasting to the Fano
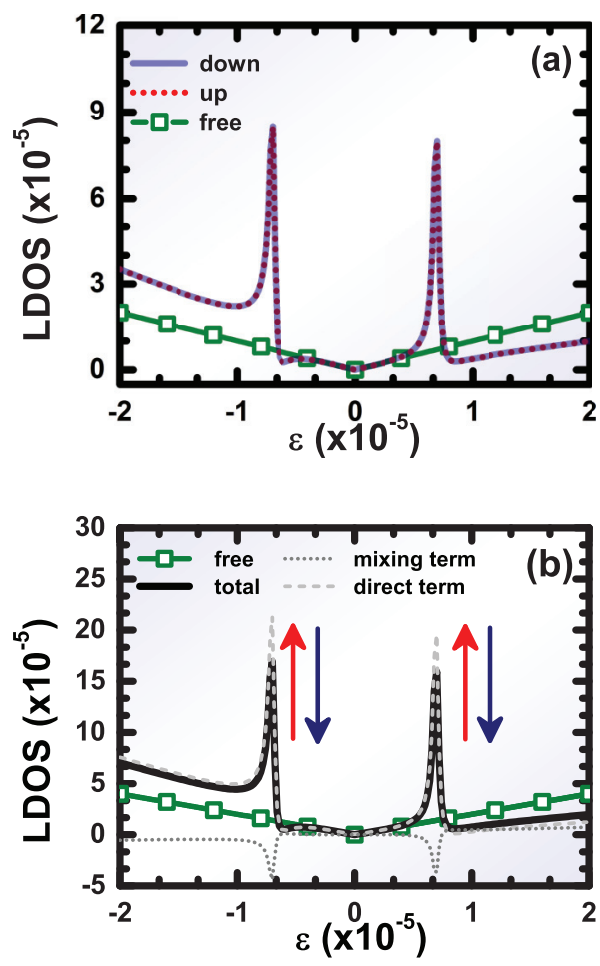

FIG. 4. (Color online) Parameters: $v_{0}=0.14, \varepsilon_{1 d}=\varepsilon_{2 d}=0$, $t_{d 1} / t_{c}=t_{d 2} / t_{c}=1 \times 10^{-5}$ (symmetric limit of Fano factors), and $\Delta_{1}=-\Delta_{2}=2 \times 10^{-5}$ (antiparallel magnetic fields). (a) In the symmetric limit, $\rho_{\text {LDOS }}^{\sigma}$ [Eq. (32)] is spin degenerate. As a result, the dotted red curve and solid blue curve for spins up and down, respectively, are superimposed. For comparison, the DOS for graphene free of impurities [Eq. (6)] is represented by the green line with squares. (b) The solid black curve represents the total LDOS given by sum of spin-up and spin-down contributions [Eq. (36)]. Arrows are included in order to illustrate the spins corresponding to the resonance. The green curve with squares is the same as in (a). Additionally, the dashed gray and dotted gray lines are the plots of Eqs. (33) and (34), respectively. These equations correspond to the direct and mixed contributions for the total LDOS under the presence of the impurities.

antiresonances found in the mixing term, which are signatures of destructive interference. As a result, the total LDOS of Eq. (36) is given by the solid black curve. The green line with squares gives $\Sigma_{\sigma} \rho^{\mathrm{GS}}(\varepsilon)$ determined by Eq. (6), which represents the DOS of the graphene system in the absence of the impurities where the peaks are absent as expected.

In Fig. 5, we analyze the asymmetric limit of the Fano factors established by the condition $t_{d 1} / t_{c} \neq t_{d 2} / t_{c}$. For this situation, with $t_{d 1} / t_{c}=1 \times 10^{-5}$ and $t_{d 2} / t_{c}=0.5 \times 10^{-5}$, we have verified that the total LDOS becomes spin dependent. Such a feature appears in Fig. 5(a), where the distinction between the up and down components of the LDOS is evident $\left(\rho_{\text {LDOS }}^{\uparrow} \neq \rho_{\text {LDOS }}^{\downarrow}\right)$. In the range of negative energies, two aligned peaks with different amplitudes exist, but the corresponding for spin down (solid blue curve) is more pronounced in respect to the spin up (dotted red curve), i.e., $\rho_{\text {LDOS }}^{\downarrow}>\rho_{\text {LDOS }}^{\uparrow}$. At positive energies, this pattern is reversed $\left(\rho_{\text {LDOS }}^{\uparrow}>\rho_{\text {LDOS }}^{\downarrow}\right)$. Thus, depending on which resonance peak is probed by the STM tip, placed at $\varepsilon \simeq-0.7 \times 10^{-5}$ or at 

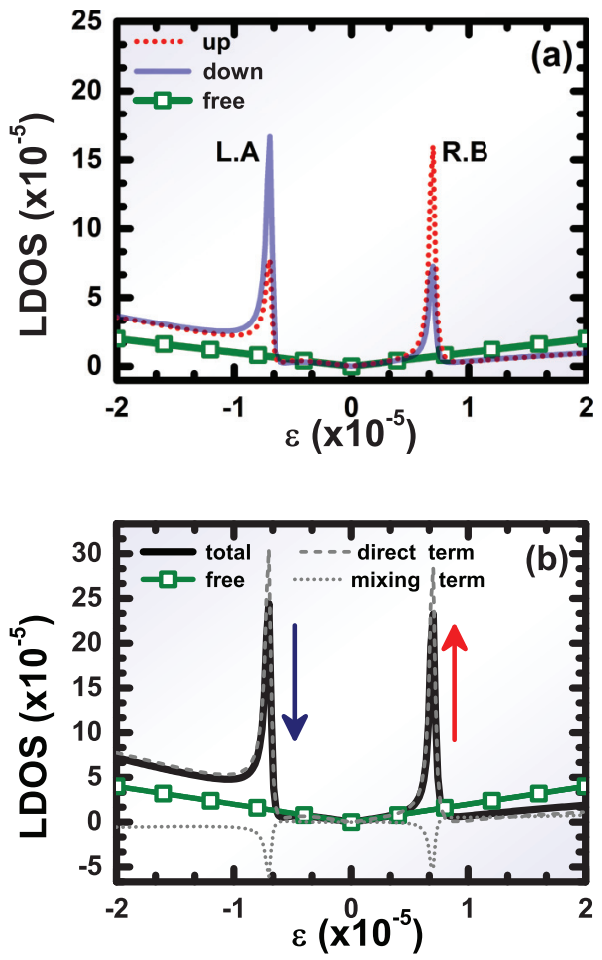

FIG. 5. (Color online) Parameters: $v_{0}=0.14, \varepsilon_{1 d}=\varepsilon_{2 d}=0$, $t_{d 1} / t_{c}=1 \times 10^{-5}$, and $t_{d 2} / t_{c}=0.5 \times 10^{-5}$ (asymmetric limit of Fano factors), with $\Delta_{1}=-\Delta_{2}=2 \times 10^{-5}$ (antiparallel magnetic fields). (a) In the asymmetric limit, $\rho_{\text {LDOs }}^{\sigma}$ [Eq. (32)] becomes spin dependent, which is evident by the peaks with different amplitudes for spin up (dotted red curve) and spin down (solid blue curve). For comparison, the DOS for graphene free of impurities [Eq. (6)] is represented by the green line with squares. (b) By summing the curves of (a) for spin up and spin down, one obtains the solid black curve for the total LDOS [Eq. (36)]. In contrast to the results of Fig. 4(b), now the total LDOS exhibits a spin polarization, as indicated by the arrows at each peak. Also shown are the dashed gray and dotted gray lines for the plots of Eqs. (33) and (34), respectively. Notice that in this case the direct contribution is stronger than the symmetric case of Fig. 4.

$\varepsilon \simeq 0.7 \times 10^{-5}$, the system filters predominantly spin down or spin up, respectively.

The origin of such a filtering lies within the direct term $\Sigma_{\sigma} \rho_{1122}^{\sigma}$ determined by Eq. (33); in particular, it arises from the contribution of $-\left(q_{T 2}^{\mathrm{GS}}\right)^{2} \operatorname{Im}\left(\tilde{\mathcal{R}}_{d_{2} d_{2}}^{\sigma}\right)=\left(q_{T 2}^{\mathrm{GS}}\right)^{2} \pi \rho^{\mathrm{GS}}(D) \operatorname{DOS}_{22}^{\sigma}$, where we have used Eq. (47). As $\left|q_{T 2}^{\mathrm{GS}}\right|>\left|q_{T 1}^{\mathrm{GS}}\right|$ is valid for the ranges out of the shaded regions (sides " $L$ " and " $R$ ") in Fig. 2(a), the parameter $\left(q_{T 2}^{\mathrm{GS}}\right)^{2}$ enhances the resonances " $\mathrm{A}$ " and "B" of Fig. 3(b), thus resulting in the peaks "L.A" and "R.B" in Fig. 5(a).

In Fig. 5(b) we have performed the same analysis as done for Fig. 4(b). We have verified that in the asymmetric limit $t_{d 1} / t_{c} \neq t_{d 2} / t_{c}$, the mixing term $\Sigma_{\sigma} \rho_{1221}^{\sigma}$ calculated from Eq. (34) (dotted gray curve) suppresses the peaks of the direct term $\Sigma_{\sigma} \rho_{1122}^{\sigma}$ determined by Eq. (33) (dashed gray curve) as well as in the symmetric regime $t_{d 1} / t_{c}=t_{d 2} / t_{c}$ of Fig. 4(b). This suppression leads to the solid black curve, which is obtained from Eq. (36). In contrast to the results of Fig. 4(b), in Fig. 5(b) each black peak of the total LDOS exhibits a finite


FIG. 6. (Color online) Parameters: $v_{0}=0.14, \varepsilon_{1 d}=\varepsilon_{2 d}=0$, $t_{d 1} / t_{c}=10 \times 10^{-5}$, and $t_{d 2} / t_{c}=0.5 \times 10^{-5}$ (asymmetric limit of Fano factors), with $\Delta_{1}=-\Delta_{2}=2 \times 10^{-5}$ (antiparallel magnetic fields). (a) In the asymmetric limit, $\rho_{\mathrm{LDOS}}^{\sigma}$ [Eq. (32)] is spin dependent, as one can observe by comparing the curves for spin up (red dotted curve) and spin down (solid blue curve). (b) The total density of states LDOS of Eq. (36) (solid black curve) and the contributions of the direct (dashed gray curve) and mixing (dotted gray curve) LDOS obtained from Eqs. (33) and (34). The arrows indicate the majority spin corresponding to the resonance. The increase of $t_{d 1} / t_{c}=10 \times 10^{-5}$ leads to the inversion of the mixing curve which exhibits resonances instead of antiresonances, as previously shown in Fig. 5(b). As a consequence, the majority spin at each peak is also inverted, in comparison to Fig. 5(b). This shows that is possible to filter either spin up or spin down by just varying the distance between the STM tip and the adatom.

polarization whose majority spin is indicated by an arrow at each peak.

In order to investigate the role of the mixing term $\Sigma_{\sigma} \rho_{1221}^{\sigma}$ upon the total LDOS, we have considered in Fig. 6 the STM tip closer to the host surface. To accomplish this situation, we have increased $t_{d 1} / t_{c}$ to $10 \times 10^{-5}$, keeping $t_{d 2} / t_{c}$ fixed to $0.5 \times 10^{-5}$. Hence, the value of $t_{d 1} / t_{c}$ is ten times greater than the corresponding value used in Fig. 5, which makes the mixing term more relevant in this case. Figure 6(a) exhibits enhanced resonances with respect to those found in Fig. 5(a). Notice that the scale of the LDOS axis is also enlarged by a factor of ten, thus the background DOS of the graphene [not displayed in Fig. 6(a)] acts as a flat band within this scale. Moreover, the main difference between Figs. 5(a) and 6(a) is the reversal of the majority spin for the resonances.

In Fig. 6(a), the peak at $\varepsilon \simeq-0.7 \times 10^{-5}$ is dominated by spin-up electrons ( $\rho_{\text {LDOS }}^{\uparrow}>\rho_{\text {LDOS }}^{\downarrow}$ in the dotted red curve), while the corresponding at $\varepsilon \simeq+0.7 \times 10^{-5}$ is due to spin 


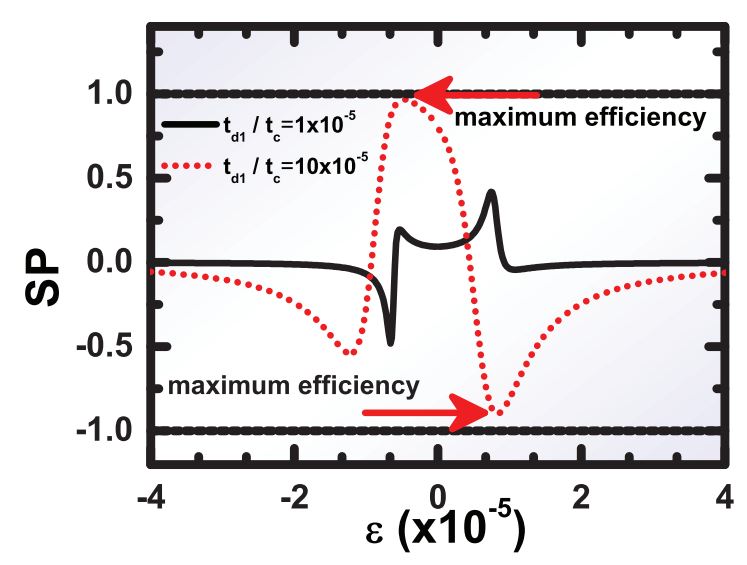

FIG. 7. (Color online) Parameters: $v_{0}=0.14, \varepsilon_{1 d}=\varepsilon_{2 d}=0$, $t_{d 2} / t_{c}=0.5 \times 10^{-5}$, and $\Delta_{1}=-\Delta_{2}=2 \times 10^{-5}$ (antiparallel magnetic fields). Transport polarization of Eq. (37) as a function of the energy $\varepsilon$. $t_{d 1} / t_{c}=1 \times 10^{-5}$ for the solid black curve. $t_{d 1} / t_{c}=$ $10 \times 10^{-5}$ for the dotted red curve. By increasing the value of $t_{d 1} / t_{c}$ to $10 \times 10^{-5}$ it is possible to obtain two points at which the efficiency reaches a maximum value. In particular, for $\varepsilon \simeq-0.5 \times 10^{-5}$ the efficiency is $100 \%$, which leads to a full polarized current through the STM system.

down $\left(\rho_{\text {LDOS }}^{\downarrow}>\rho_{\text {LDOS }}^{\uparrow}\right.$ in the solid blue curve). For Fig. 5(a), we have exactly the opposite. The origin lies within the term $-\left(q_{T 1}^{\mathrm{GS}}\right)^{2} \operatorname{Im}\left(\tilde{\mathcal{R}}_{d_{1} d_{1}}^{\sigma}\right)=\left(q_{T 1}^{\mathrm{GS}}\right)^{2} \pi \rho^{\mathrm{GS}}(D) \mathrm{DOS}_{11}^{\sigma}$.

In the case of Fig. 6(a), the corresponding Fano parameters are described by the curves in Fig. 2(b) in which $\left|q_{T 1}^{\mathrm{GS}}\right|>\left|q_{T 2}^{\mathrm{GS}}\right|$ for the whole energy range [shaded regions "LL" and "RR" in Fig. 2(b)]. Thus, the peaks "AA" and "BB" of Fig. 3(a) are enhanced by $\left(q_{T 1}^{\mathrm{GS}}\right)^{2}$ and lead to the new resonances "LL.AA" and "RR.BB" in Fig. 6(a).

We point out that not only does the spin-filtering effect becomes reversed, but also does the Fano interference arising from $\Sigma_{\sigma} \rho_{1221}^{\sigma}$ in Eq. (34). Figure 6(b) shows that the mixing term (dotted gray curve) is formed by a pair of resonances, which is the opposite pattern as compared to that found in Figs. 4(b) and 5(b). It means that the destructive interference is replaced by a constructive one. As a result, the direct and mixed terms (dashed and dotted gray curves, respectively) are now being summed, leading to the total LDOS [Eq. (36)] represented by the solid black curve. Once again, we have used an arrow at each peak to denote the down or up majority spin. In summary, the lifting of the spin degeneracy in the "host + impurities" device is not established by the Zeeman effect $\Delta_{1}=-\Delta_{2}$, but is due to the asymmetric ratios $t_{d 1} / t_{c} \neq t_{d 2} / t_{c}$. Our work proposes the Fano interference as the mechanism to filter spins in graphene. Additionally, our spin filter exhibits the opposite behavior of that for a single adatom, ${ }^{6}$ in which the spin-polarized feature of the system is quenched by the proximity of the STM tip.

The degree of spin polarization for the transport through the considered setup given by Eq. (37) as a function of the energy $\varepsilon$ is displayed in Fig. 7. We have analyzed the cases $t_{d 1} / t_{c}=$ $1 \times 10^{-5}$ (solid black curve) and $t_{d 1} / t_{c}=10 \times 10^{-5}$ (dotted red curve). In the former situation, for energies below and near the Fermi level, values of positive polarizations $\left(\mathcal{G}^{\uparrow}>\mathcal{G}^{\downarrow}\right)$ and negative $\left(\mathcal{G}^{\downarrow}>\mathcal{G}^{\uparrow}\right)$ occur, while above the Fermi level,

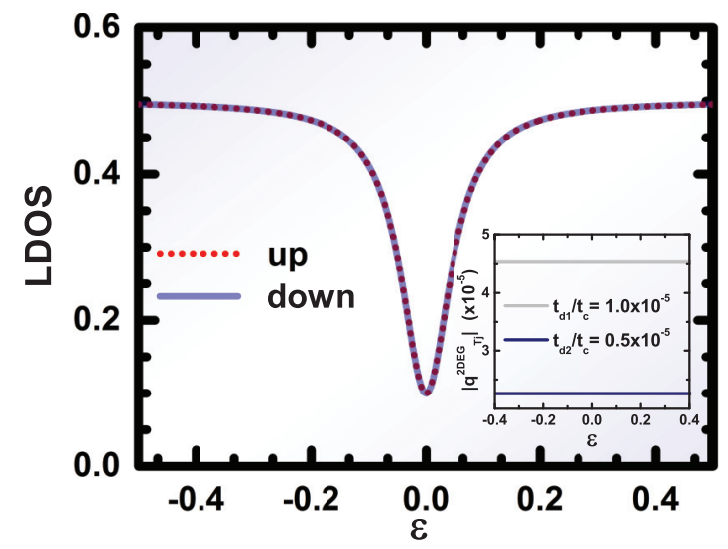

FIG. 8. (Color online) Parameters: $v_{0}=0.14, \varepsilon_{1 d}=\varepsilon_{2 d}=0$, $t_{d 1} / t_{c}=1 \times 10^{-5}, t_{d 2} / t_{c}=0.5 \times 10^{-5}$, and $\Delta_{1}=-\Delta_{2}=2 \times 10^{-5}$ (antiparallel magnetic fields). The $\rho_{\text {LDOS }}^{\sigma}$ [Eq. (32)] is presented as a function of the energy $\varepsilon$ for the 2DEG. Since the curves for spin up (dotted red curve) and spin down (solid blue curve) are superimposed, the total LDOS does not depend on spin, as observed for the graphene sheet. Hence, the 2DEG system does not operate as a Fano spin filter. Unlike the graphene, in the 2DEG we always have $\left|q_{T 1}^{2 \mathrm{DEG}}\right|>\left|q_{T 2}^{2 \mathrm{DEG}}\right|$ for $t_{d 1} / t_{c}>t_{d 2} / t_{c}$ (inset).

polarization remains positive. However, it never exceeds $|0.5|$. In the case $t_{d 1} / t_{c}=10 \times 10^{-5}$, which mimics the STM tip closer to the host, the pattern of the polarization observed for $t_{d 1} / t_{c}=1 \times 10^{-5}$ is reversed. Moreover, it reaches the maximum value +1 at $\varepsilon \simeq-0.5 \times 10^{-5}$ and approaches -1 for $\varepsilon \simeq+0.8 \times 10^{-5}$ (see the horizontal arrows).

\section{B. 2DEG system}

In this section we explore the 2DEG system with standard quadratic dispersion. To this end, we employ Eq. (36) for the LDOS by taking into account Eqs. (7), (45), (46), (50), (54), and (55).

Figure 8 reveals that even in the asymmetric limit, for which $t_{d 1} / t_{c}=1 \times 10^{-5}$ and $t_{d 2} / t_{c}=0.5 \times 10^{-5}$, there is no resolved spin dependence in the Fano profile of the LDOS. Such a feature can be visualized via the dotted red and solid blue curves, which are characterized by degenerate antiresonances for spins up and down, respectively. These resonances are predictable due to Eq. (54) for the Fano parameter, which gives $\left|q_{T j}^{2 \mathrm{DEG}}\right| \rightarrow 0$ for $\varepsilon \rightarrow 0$ (see the "gray" and "blue" line shapes in the inset, respectively, for $t_{d 1} / t_{c}=1 \times 10^{-5}$ and $t_{d 2} / t_{c}=$ $\left.0.5 \times 10^{-5}\right)$. It is worth noting that for the $2 \mathrm{DEG}$, unlike the graphene setup, there is no region in the Fano versus $\varepsilon$ plot where the condition $\left|q_{T 2}^{2 \mathrm{DEG}}\right|>\left|q_{T 1}^{2 \mathrm{DEG}}\right|$ for $t_{d 1} / t_{c}>t_{d 2} / t_{c}$ is verified (compare the inset with the results in Fig. 2). As a result, the 2DEG setup does not operate as a spin filter.

\section{CONCLUSIONS}

In this paper, we have proposed a relativistic spin filter consisting of an STM setup with a graphene hosting two lateral impurities. The mechanism through which the STM picks up preferentially a definite spin is based on quantum Fano interference. A particular feature provided by this system is the possibility to choose which spin to filter by tuning the distance 
between the STM tip and the adatom. For particular conditions pure spin currents may be generated by the proposed setup, which makes it attractive for possible spintronics applications.

It is worth mentioning that the subsurface impurity plays an important role on the transport properties in spite of the weak coupling to the STM tip. In fact, in Fig. 2 there are regions in which the condition $\left|q_{T 2}^{\mathrm{GS}}\right|>\left|q_{T 1}^{\mathrm{GS}}\right|$ is verified even with $t_{d 1} / t_{c}>t_{d 2} / t_{c}$. This is a striking result of quantum interference and illustrates the subtle quantum properties of graphene based structures. These results contrast with $2 \mathrm{DEG}$, where the Fano factors always follow the same trend, i.e., $\left|q_{T 1}^{2 \mathrm{DEG}}\right|>\left|q_{T 2}^{2 \mathrm{DEG}}\right|$ when $t_{d 1} / t_{c}>t_{d 2} / t_{c}$.

\section{ACKNOWLEDGMENT}

This work has the support of the Brazilian agencies CNPq, CAPES, and PROPG-PROPe/UNESP, and FP7 IRSES project SPINMET. A. C. Seridonio thanks the University of Iceland for hospitality.
${ }^{1}$ K. S. Novoselov, Rev. Mod. Phys. 83, 837 (2011)

${ }^{2}$ S. Das Sarma, S. Adam, E. H. Hwang, and E. Rossi, Rev. Mod. Phys. 83, 407 (2011).

${ }^{3}$ N. M. R. Peres, Rev. Mod. Phys. 82, 2673 (2010).

${ }^{4}$ A. H. Castro Neto, F. Guinea, N. M. R. Peres, K. S. Novoselov, and A. K. Geim, Rev. Mod. Phys. 81, 109 (2009).

${ }^{5}$ B. Uchoa, V. N. Kotov, N. M. R. Peres, and A. H. Castro Neto, Phys. Rev. Lett. 101, 026805 (2008).

${ }^{6}$ B. Uchoa, L. Yang, S.-W. Tsai, N. M. R. Peres, and A. H. Castro Neto, Phys. Rev. Lett. 103, 206804 (2009).

${ }^{7}$ Z. G. Zhu and J. Berakdar, Phys. Rev. B 84, 165105 (2011).

${ }^{8}$ M. P. Lima, A. J. R. da Silva, and A. Fazzio, Phys. Rev. B 84, 245411 (2011).

${ }^{9}$ V. W. Brar, R. Decker, H. M. Solowan, Y. Wang, L. Maserati, K. T. Chan, H. Lee, Ç. O. Girit, A. Zettl, S. G. Louie, M. L. Cohen, and M. F. Crommie, Nat. Phys. 7, 43 (2011).

${ }^{10}$ A. Saffarzadeh and G. Kirczenow, Phys. Rev. B 85, 245429 (2012).

${ }^{11}$ F. Hiebel, P. Mallet, J. Y. Veuillen, and L. Magaud, Phys. Rev. B 86, 205421 (2012).

${ }^{12}$ P. S. Cornaglia, G. Usaj, and C. A. Balseiro, Phys. Rev. Lett. 102, 046801 (2009).

${ }^{13}$ T. Eelbo, M. Wasniowska, M. Gyamfi, S. Forti, U. Starke, and R. Wiesendanger, Phys. Rev. B 87, 205443 (2013).

${ }^{14}$ T. Eelbo, M. Wasniowska, P. Thakur, M. Gyamfi, B. Sachs, T. O. Wehling, S. Forti, U. Starke, C. Tieg, A. I. Lichtenstein, and R. Wiesendanger, Phys. Rev. Lett. 110, 136804 (2013).

${ }^{15}$ T. P. Hardcastle, C. R. Seabourne, R. Zan, R. M. D. Brydson, U. Bangert, Q. M. Ramasse, K. S. Novoselov, and A. J. Scott, Phys. Rev. B 87, 195430 (2013).

${ }^{16}$ Y. Virgus, W. Purwanto, H. Krakauer, and S. Zhang, Phys. Rev. B 86, 241406 (2012).

${ }^{17}$ A. N. Rudenko, F. J. Keil, M. I. Katsnelson, and A. I. Lichtenstein, Phys. Rev. B 86, 075422 (2012).

${ }^{18}$ K. T. Chan, J. B. Neaton, and M. L. Cohen, Phys. Rev. B 77, 235430 (2008).

${ }^{19}$ B. Huang, J. Yu, and S.-H. Wei, Phys. Rev. B 84, 075415 (2011).

${ }^{20}$ X. Hong, S.-H. Cheng, C. Herding, and J. Zhu, Phys. Rev. B 83, 085410 (2011).

${ }^{21}$ P. O. Lehtinen, A. S. Foster, A. Ayuela, A. Krasheninnikov, K. Nordlund, and R. M. Nieminen, Phys. Rev. Lett. 91, 017202 (2003).

${ }^{22}$ J. H. Chen, C. Jang, S. Adam, M. S. Fuhrer, E. D. Williams, and M. Ishigami, Nat. Phys. 4, 377 (2008).
${ }^{23}$ K. Pi, K. M. McCreary, W. Bao, W. Han, Y. F. Chiang, Y. Li, S.-W. Tsai, C. N. Lau, and R. K. Kawakami, Phys. Rev. B 80, 075406 (2009).

${ }^{24}$ M. Alemani, A. Barfuss, B. Geng, C. Girit, P. Reisenauer, M. F. Crommie, F. Wang, A. Zettl, and F. Hellman, Phys. Rev. B 86, 075433 (2012)

${ }^{25}$ F. Schedin, A. Geim, S. Morozov, E. Hill, P. Blake, M. Katsnelson, and K. Novoselov, Nat. Mater. 6, 652 (2007).

${ }^{26}$ T. O. Wehling, K. S. Novoselov, S. V. Morozov, E. E. Vdovin, M. I. Katsnelson, A. K. Geim, and A. I. Lichtenstein, Nano Lett. 8, 173 (2008).

${ }^{27}$ M. Gyamfi, T. Eelbo, M. Wasniowska, T. O. Wehling, S. Forti, U. Starke, A. I. Lichtenstein, M. I. Katsnelson, and R. Wiesendanger, Phys. Rev. B 85, 161406 (2012).

${ }^{28}$ M. Gyamfi, T. Eelbo, M. Was'niowska, and R. Wiesendanger, Phys. Rev. B 84, 113403 (2011).

${ }^{29}$ J. Tersoff and D. R. Hamann, Phys. Rev. Lett. 50, 1998 (1983).

${ }^{30}$ J. Tersoff and D. R. Hamann, Phys. Rev. B 31, 805 (1985).

${ }^{31}$ U. Fano, Phys. Rev. 124, 1866 (1961).

${ }^{32}$ A. E. Miroshnichenko, S. Flach, and Y. S. Kivshar, Rev. Mod. Phys. 82, 2257 (2010).

${ }^{33}$ A. C. Hewson, The Kondo Problem to Heavy Fermions (Cambridge University Press, Cambridge, UK, 1993).

${ }^{34}$ H. C. Manoharan, C. P. Lutz, and D. M. Eigler, Nature (London) 403, 512 (2000).

${ }^{35}$ V. Madhavan, W. Chen, T. Jamneala, M. F. Crommie, and N. S. Wingreen, Phys. Rev. B 64, 165412 (2001).

${ }^{36}$ N. Knorr, M. A. Schneider, L. Diekhöner, P. Wahl, and K. Kern, Phys. Rev. Lett. 88, 096804 (2002).

${ }^{37}$ A. F. Otte, M. Ternes, K. V. Bergmann, S. Loth, H. Brune, C. P. Lutz, C. F. Hirjibehedin, and A. J. Heinrich, Nat. Phys. 4, 847 (2008).

${ }^{38}$ M. Ternes, A. J. Heinrich, and W. D. Schneider, J. Phys.: Condens. Matter 21, 053001 (2009).

${ }^{39}$ A. C. Seridonio, F. S. Orahcio, F. M. Souza, and M. S. Figueira, Phys. Rev. B 85, 165109 (2012).

${ }^{40}$ S. L. Kawahara, J. Lagoute, V. Repain, C. Chacon, Y. Girard, J. Klein, and S. Rousset, Phys. Rev. B 82, 020406(R) (2010).

${ }^{41}$ A. C. Seridonio, F. M. Souza, and I. A. Shelykh, J. Phys.: Condens. Matter 21, 095003 (2009).

${ }^{42}$ A. C. Seridonio, F. M. Souza, J. Del Nero, and I. A. Shelykh, Physica E 41, 1611 (2009).

${ }^{43}$ P. H. Penteado, F. M. Souza, A. C. Seridonio, E. Vernek, and J. C. Egues, Phys. Rev. B 84, 125439 (2011). 
${ }^{44}$ N. Néel, J. Kröger, and R. Berndt, Phys. Rev. B 82, 233401 (2010).

${ }^{45}$ Y. Qi, J. X. Zhu, S. Zhang, and C. S. Ting, Phys. Rev. B 78, 045305 (2008).

${ }^{46}$ M. Sindel, L. Borda, J. Martinek, R. Bulla, J. König, G. Schön, S. Maekawa, and J. von Delft, Phys. Rev. B 76, 045321 (2007).

${ }^{47}$ K. Hamaya, M. Kitabatake, K. Shibata, M. Jung, M. Kawamura, K. Hirakawa, T. Machida, and T. Taniyama, Appl. Phys. Lett. 91, 232105 (2007).

${ }^{48}$ K. Hamaya, M. Kitabatake, K. Shibata, M. Jung, M. Kawamura, S. Ishida, T. Taniyama, K. Hirakawa, Y. Arakawa, and T. Machida, Phys. Rev. B 77, 081302(R) (2008).

${ }^{49}$ M. R. Calvo, J. F. Rossier, J. J. Palacios, D. Jacob, D. Natelson, and C. Untiedt, Nature (London) 458, 1150 (2009).

${ }^{50}$ J. Hauptmann, J. Paaske, and P. Lindelof, Nat. Phys. 4, 373 (2008).

${ }^{51}$ I. Weymann and L. Borda, Phys. Rev. B 81, 115445 (2010).

${ }^{52}$ I. Weymann, Phys. Rev. B 83, 113306 (2011).

${ }^{53}$ M. Misiorny, I. Weymann, and J. Barnaś, Phys. Rev. Lett. 106, 126602 (2011).

${ }^{54}$ M. Gaass, A. K. Hüttel, K. Kang, I. Weymann, J. von Delft, and Ch. Strunk, Phys. Rev. Lett. 107, 176808 (2011).
${ }^{55}$ M. Misiorny, I. Weymann, and J. Barnaś, Phys. Rev. B 84, 035445 (2011).

${ }^{56}$ C. A. Büsser, A. E. Feiguin, and G. B. Martins, Phys. Rev. B 85, 241310(R) (2012).

${ }^{57}$ E. Vernek, C. A. Büsser, E. V. Anda, A. E. Feiguin, and G. B. Martins, arXiv:1308.4746.

${ }^{58}$ M. E. Torio, K. Hallberg, S. Flach, A. E. Miroshnichenko, and M. Titov, Eur. Phys. J. B 37, 399 (2004).

${ }^{59}$ P. W. Anderson, Phys. Rev. 124, 41 (1961).

${ }^{60}$ D. Goldhaber-Gordon, H. Shtrikman, D. Mahalu, D. AbuschMagder, U. Meirav, and M. A. Kastner, Nature (London) 391, 156 (1998).

${ }^{61}$ S. M. Cronenwett, T. H. Oosterkamp, and L. P. Kouwenhoven, Science 281, 540 (1998).

${ }^{62} \mathrm{H}$. Haug and A. P. Jauho, Quantum Kinetics in Transport and Optics of Semiconductors, Springer Series in Solid-State Sciences Vol. 123 (Springer, New York, 1996).

${ }^{63}$ M. Plihal and J. W. Gadzuk, Phys. Rev. B 63, 085404 (2001).

${ }^{64}$ C.-Y. Lin, A. H. Castro Neto, and B. A. Jones, Phys. Rev. Lett. 97, 156102 (2006). 